\title{
Tratamento do transtorno
}

\section{bipolar - Eutimia}

Bipolar Disorder Treatment - Euthymia

\section{Fábio Gomes de Matos e Souza ${ }^{1}$ Resumo}

\begin{abstract}
O transtorno bipolar é um quadro complexo caracterizado por episódios de depressão, mania ou hipomania e fases assintomáticas. O tratamento visa ao controle de episódios agudos e prevenção de novos episódios. O tratamento farmacológico iniciou-se com o lítio. Até o momento, o lítio permanece como o tratamento com mais evidências favoráveis na fase de manutenção. Outros tratamentos demonstram eficácia nessa fase, como o valproato, a carbamazepina e os antipsicóticos atípicos. Dos antipsicóticos atípicos o mais estudado nesta fase do tratamento é a olanzapina. Mais estudos prospectivos são necessários para confirmar a ação profilática de novos agentes.
\end{abstract}

Palavras-chave: Transtorno bipolar, manutenção, estabilizadores do humor.

\begin{abstract}
Bipolar disorder is a complex disorder characterized by depression episodes, mania or hypomania and asymptomatic phases. The treatment aims at the control of acute episodes and prevention of new episodes. The pharmacological treatment was inaugurated with lithium. Until the moment, lithium remains as the treatment with more favorable evidences in the maintenance phase. Other treatments demonstrate efficacy in this phase, as valproate, carbamazepine and atypical antipsychotics. Of the atypical antipsychotics, the most studied in this phase of treatment is olanzapine. More prospective studies are necessary to confirm prophylactic action of new agents.
\end{abstract}

Key words: Bipolar disorder, maintenance, mood stabilizers.

O transtorno bipolar é um transtorno crônico e complexo caracterizado por episódios de depressão, mania ou hipomania de forma isolada ou mista com grande morbidade e mortalidade. O tratamento do paciente eutímico deve sempre considerar a possibilidade de o paciente vir a ter episódios de mania e/ou depressão. Aeutimia, usualmente, é definida como a remissão dos sintomas, entretanto, idealmente, seria o período no qual o paciente não apenas estaria sem sintomas, mas (re)integrado funcionalmente em suas atividades de rotina. O objetivo do tratamento, portanto, é manter o paciente sem sintomas. Assim, a meta principal do tratamento é a remissão e não apenas a resposta clínica (redução de 50\% dos sintomas observados), que 
é comumente usada como medida de desfecho nos ensaios clínicos.

O tratamento do transtorno bipolar é dividido em três fases: aguda, continuação e manutenção. Os objetivos do tratamento da fase aguda são: tratar mania sem causar depressão e/ou consistentemente melhorar depressão sem causar mania. A fase de continuação tem como meta: estabilizar os benefícios, reduzir os efeitos colaterais, tratar até a remissão, reduzir a possibilidade de recaída e aumentar o funcionamento global. Finalmente, os objetivos do tratamento de manutenção são: prevenir mania e/ou depressão e maximizar recuperação funcional, ou seja, que o paciente continue em remissão.

O surgimento de sintomas na fase de continuação constitui-se em uma recaída enquanto que, se acontecesse na fase de manutenção, seria uma recorrência. Embora na maior parte do tempo o tratamento clínico do transtorno bipolar ocorra durante a fase de manutenção, existem poucas evidências empíricas para orientar que drogas deveriam ser utilizadas para manter a eutimia. Quando o paciente responde ao tratamento instituído na fase aguda, as mesmas drogas usualmente continuam a ser utilizadas no tratamento profilático.

No caso do transtorno bipolar, o que acontece com os pacientes após o episódio maníaco e/ou depressivo ter sido controlado no ensaio clínico que aborda eficácia não tem recebido a atenção desejada em termos de acompanhamento sistemático na literatura.

O uso de antidepressivos e antipsicóticos na fase de manutenção do transtorno bipolar é bastante comum na prática clínica, entretanto a literatura contém poucos dados sobre a eficácia e segurança dessas drogas no tratamento de longo prazo desses pacientes.

Esta revisão basear-se-á na melhor evidência disponível, principalmente em revisões sistemáticas e ensaios clínicos controlados. Entretanto, muitas questões relativas ao tratamento da fase de manutenção ainda não foram respondidas por meio de estudos randomizados com o nível de segurança científica adequado. Portanto, quando não existirem estudos com maior poder científico, opiniões de experts e estudos abertos serão usados como referência. Os únicos ensaios clínicos controlados envolvem pacientes com transtorno bipolar tipo I (Sachs e Thase, 2000a). Dados sobre subtipos de pacientes, como bipolares tipo II e cicladores rápidos, são raros na literatura.

Em muitos ensaios clínicos, há uma ênfase excessiva em significância estatística em detrimento do que aquele achado representa em termos de melhora clínica do paciente. Assim, uma redução de um ou dois pontos em uma escala pode ser extremamente significativa em termos estatísticos, mas apenas representando uma pequena melhora do estado geral do paciente. Assim, a leitura dos dados aqui apresentados deve ser contextualizada dentro desse prisma.
O ideal da medicina baseada em evidências seria que a decisão clínica fosse a união da evidência baseada em pesquisa mais os fatores individuais do paciente. Entretanto, outros fatores influenciam na decisão clínica da prescrição medicamentosa: experiência prática com a droga; doses mais fáceis de serem administradas; monitoramento do tratamento mais fácil ou difícil; custos do medicamento; familiaridade com a droga e, não menos importante, propaganda intensiva dos laboratórios.

\section{Pontos a considerar no tratamento de manutenção}

\section{Adesão}

Uma metanálise de 19 estudos com 546 indivíduos que apresentaram descontinuação do lítio demonstrou que o tempo para recaída era de três meses em $50 \%$ dos indivíduos. A recaída era usualmente maníaca, e os bipolares tipo I eram mais prováveis de recair. Assim, o tratamento deve objetivar a adesão dos pacientes como forma de eles se manterem eutímicos (Baker, 1994). Em conseqüência, os pacientes devem ser advertidos sobre o risco de recorrência por ocasião da descontinuação da medicação.

\section{Comorbidades}

É importante também observar que tipo de comorbidade o paciente apresenta para escolher a droga que melhor se adapta ao seu perfil. Dependência química, diferentes transtornos de ansiedade, transtorno do déficit de atenção e hiperatividade são entidades clínicas comórbidas freqüentes do transtorno bipolar.

\section{Efeitos colaterais}

Os efeitos colaterais das drogas são o principal fator de não-adesão (Fagiolini et al., 2003). Assim, deve haver monitoramento dos parâmetros basais e, ao longo do tempo, para identificar e tratar precocemente os efeitos colaterais dos pacientes em risco. Para drogas que aumentam o peso, por exemplo, mudanças nos níveis de glicose e lipídeos devem ser observadas.

\section{Suicídio}

A gravidade da depressão bipolar e o risco de o paciente bipolar cometer suicídio é alta. A taxa de prevalência de suicídio comparada à taxa observada na população é em geral 30 vezes maior (Jamison, 2000; MullerOerlinghausen, 2001).

\section{Uso da psicoterapia/educação}

A psicoterapia pode reduzir os fatores de risco de recorrência (paciente permanecendo eutímico), melho- 
rando a adesão, propiciando as mudanças no estilo de vida, permitindo a detecção precoce de sintomas prodrômicos e melhor gerenciamento de dificuldades interpessoais (Miklowitz et al., 2000; Rothbaum e Astin, 2000).

Os principais objetivos do tratamento psicoterápico no transtorno bipolar, segundo Perry et al. (1999), são:

- adesão - é fundamental aumentar a adesão do paciente ao tratamento;

- funcionamento social e ocupacional - melhorar o desempenho dos pacientes em atividades sociais e laborativas;

- melhorar a detecção de sinais precoces de recorrências;

- educar o paciente acerca de sua doença e suas medicações (explicar os vários sintomas, esclarecer sobre prognóstico, instalar esperança, envolver familiares);

- promover um estilo de vida saudável, por exemplo, regularizar o ciclo sono-vigília;

- criar de forma colaborativa estratégias de lidar com estresses, que, se não administrados apropriadamente, podem provocar um episódio depressivo.

Existem evidências de que vários tipos de psicoterapia podem ajudar os pacientes a permanecerem eutímicos. A terapia cognitiva tem ajudado vários pacientes (Scott et al., 2001) bem como a terapia familiar (Miklowitz, 1997). Terapia de grupo pode também melhorar a adesão e a auto-estima (Colom et al., 2003). Grupos de suporte fornecem informações sobre o transtorno bipolar e seu tratamento para pacientes e familiares.

\section{Uso de estabilizadores do humor}

Os critérios para definir um estabilizador do humor são: ser eficaz na mania e em estados mistos, tratar depressão aguda bipolar, reduzir a freqüência e/ou gravidade de recorrências maníacas e/ou depressivas, não piorar mania ou depressão ou induzir mudança ou ciclagem rápida (Bowden et al., 1997).

\section{Lítio}

O lítio é o agente que possui mais evidências como tratamento de manutenção no transtorno bipolar, prevenindo recaídas de mania mais freqüentemente que de depressão (Bowden et al., 2003). Entretanto, o lítio é também um antidepressivo (Goodwin et al., 1992). Ele ainda reduz o risco de suicídio. Em uma metanálise, o lítio demonstrou diminuir o risco de o paciente cometer suicídio em 8,85\% (95\% IC: 4,1 a 19,1) (Tondo et al., 2001).

Os pacientes para os quais o lítio é preferido são aqueles com doença hepática, no estágio final da gravidez e jovens adolescentes (Sachs et al., 2000).

Uma revisão da Cochrane envolvendo nove estudos concluiu que o lítio é mais eficaz do que o placebo em prevenir todas as recaídas dos pacientes com transtorno bipolar - $61 \%$ com placebo e $33 \%$ com lítio (Burgess et al., CDRS 2001).

\section{Valproato}

O valproato foi a primeira droga, após o lítio, a demonstrar que tem efeito no tratamento de manutenção do transtorno bipolar.

Em um estudo, embora os tratamentos (valproato, lítio e placebo) não tenham mostrado diferença estatisticamente significativa no tempo para ocorrer uma recorrência, o valproato revelou ter efeito profilático melhor que o lítio em medidas secundárias (maior tempo de duração bem-sucedida de profilaxia e menor deterioração de sintomas depressivos) e melhor que o placebo com taxas menores de descontinuação. Efeito profilático parece ser maior contra os episódios maníacos do que contra os episódios depressivos (Bowden et al., 2000).

$\mathrm{O}$ efeito positivo do valproato em remitir um episódio de mania pode ser um bom preditor para a resposta profilática (Bowden et al., 2000).

Outros estudos demonstram que o valproato sozinho ou em combinação tem eficácia equivalente ao lítio e possivelmente maior que a carbamazepina (Davis et al., 1999).

Os pacientes para os quais o valproato é preferido são aqueles com doenças cardíacas, renais, TCE, idosos com demência e dependência química (Sachs et al., 2000).

\section{Carbamazepina}

Uma série de estudos já foi publicada relacionando a carbamazepina (CBZ) e a oxcarbamazepina com profilaxia do transtorno bipolar (Lusznat et al., 1988; Coxhead et al., 1992).

O lítio mostrou-se melhor que a carbamazepina em morbidade interepisódica, taxas de desistência e de re-hospitalização (Kleindienst e Greil, 2002). Em quatro estudos, a carbamazepina foi comparada com o lítio em tratamento de manutenção do transtorno bipolar (Placidi et al., 1986; Watkins et al., 1987; Lusznat et al., 1988; Coxhead et al., 1992). Em apenas um estudo o lítio foi superior (Watkins et al., 1987), nos outros três estudos, as drogas obtiveram resultado semelhante (Keck et al.,1998). Carbamazepina é indicada como tratamento no início da doença e com um padrão alternativo do humor. Uma comparação de dez estudos de carbamazepina com o lítio mostrou uma eficácia semelhante (Davis et al., 1999). Um estudo mostra que apenas $8 \%$ dos pacientes tratados com carbamazepina ficaram estáveis por três a quatro anos (Post et al., 1990).

A carbamazepina tem potencial de interação importante com outras drogas, por isso ela vem sendo substituída pela oxcarbamazepina. Esta última tem uma série de vantagens sobre a carbamazepina: não é necessário monitorar os níveis plasmáticos, realizar 
testes de funções hepáticas nem testes sangüíneos, e causa menos indução do citocromo $\mathrm{P} 450$.

\section{Lamotrigina}

A lamotrigina demonstrou ser tão eficaz quanto o lítio no tratamento de manutenção dos pacientes com transtorno bipolar (Bowden et al., 2003). Tem algumas vantagens em seu uso: não apresenta ganho de peso nem déficit cognitivo, não é necessário controlar níveis sangüíneos. Tem eficácia no tratamento de recaídas depressivas (Ernst et al., 2003). Possui eficácia mínima em mania.

Ela também tem demonstrado resultados favoráveis na manutenção de pacientes bipolares tipo II e ciclagem rápida (Calabrese et al., 2000). A monoterapia da lamotrigina foi significativamente mais eficaz do que o placebo na depressão bipolar I (Calabrese et al., 1999).

\section{Topiramato}

Cinco estudos negativos controlados por placebo falharam. É útil como medicamento adjuvante e na redução de peso (Chengappa et al., 2001). Não é usado como droga de primeira linha para tratamento de manutenção do transtorno bipolar. Em um estudo com 76 pacientes uma melhora leve foi vista em $47 \%$ e moderada em $13 \%$ de bipolares tratados com topiramato (Ghaemi et al., 2001).

\section{Gabapentina}

Dois ensaios clínicos controlados por placebo falharam em demonstrar um desfecho clínico favorável. Não é usado como droga de primeira linha para tratamento de manutenção do transtorno bipolar (Letterman e Markowitz, 1999).

Um estudo naturalístico em pacientes com transtorno bipolar resistente produziu 18 respostas positivas com gabapentina (Schaffer e Schaffer, 1997). A gabapentina foi considerada eficaz em 30\% dos pacientes com transtorno bipolar ou unipolar (Ghaemi et al., 1998). Em um estudo com a lamotrigina, a gabapentina não foi melhor que o placebo (Obrocea et al., 2002).

\section{Outras drogas}

Metilfenidato - Mostrou-se eficaz em 11 de 14 pacientes com depressão bipolar (El-Mallakh, 2000).

Ômega-3 - Os ácidos ômega-3 produziram uma remissão significativa mais longa do que o placebo em alguns pacientes bipolares (Stoll et al., 1999).

Pramipexole - Há relatos de pacientes com depressão bipolar resistente em que a introdução da pramipexole produziu uma melhora (Goldberg et al., 1999).

Tiabagina - Tiabagina pode ser uma droga útil como terapia adjuntiva do transtorno bipolar, conti- nuando ser eficaz por diversos meses (Schaffer e Schaffer, 1999).

Bloqueadores de canais de cálcio (verapamil, nimodipina, diltiazeno) - Embora tenham demonstrado alguma eficácia no tratamento de transtorno bipolar, estas drogas não são usadas como medicamentos de primeira linha para tratamento de manutenção (Giannini et al. 1987).

\section{Uso de antipsicóticos}

Enquanto a eficácia dos antipsicóticos como tratamento de manutenção do transtorno bipolar não tenha sido sistematicamente estudada, estas drogas têm sido comumente utilizadas. Até 1998, não havia nenhum ensaio randomizado controlado que comparasse antipsicóticos com os estabilizadores na manutenção do transtorno bipolar (Tohen e Zarate, 1998).

\section{Típicos}

Embora sejam eficazes no tratamento de mania, não demonstraram eficácia em tratar depressão bipolar e têm pouca relevância no tratamento de manutenção. Os antipsicóticos típicos não foram usados com a indicação acima em virtude dos efeitos colaterais importantes observados ao longo do tempo como discinesia tardia, ganho de peso, sedação e disfunção sexual que levam à não-adesão (Esparon et al., 1986) .

\section{Atípicos}

Ensaios clínicos duplos-cegos randomizados com antipsicóticos atípicos no tratamento de manutenção do transtorno bipolar são escassos na literatura. Entretanto, existem evidências para alguns antipsicóticos atípicos.

\section{Clozapina}

Pacientes em fase maníaca ou psicótica do transtorno bipolar ou esquizoafetivo responderam significativamente melhor que os pacientes com síndromes depressivas graves (unipolar, bipolar ou esquizoafetivo) ( $\mathrm{Za}^{-}$ rate et al., 1995). O problema de agranulocitose, entre outros efeitos colaterais, impede o uso mais generalizado da clozapina. Esta tem sido usada com sucesso no tratamento de transtorno bipolar refratário (Suppes et al., 1999).

\section{Risperidona}

Existe evidência no tratamento do episódio de mania, mas não há dados em depressão bipolar. Em doses elevadas comporta-se como antipsicótico típico, incluído como efeito colateral o aumento de prolactina (Ghaemi e Sachs, 1997).

\section{Olanzapina}

Embora alguns pesquisadores acreditem que não há papel da olanzapina como monoterapia no transtorno 
bipolar (Lund et al., 2000), existem evidências que apontam papel positivo da olanzapina na manutenção do transtorno bipolar.

Seis ensaios clínicos com 1.422 participantes foram incluídos nessa revisão, havia uma alta taxa de recaída de falha em completar o tratamento em todas as drogas, o que pode ter viciado as estimativas de eficácia relativa (Bhana e Perry, 2001).

A olanzapina sozinha ou em associação com fluoxetina tem demonstrado eficácia em controlar a depressão bipolar (Tohen et al., 2002). Os pacientes que usaram olanzapina adicionada a lítio ou valproato em comparação a aqueles que usaram apenas lítio ou valproato levaram mais tempo para ter recaídas (Tohen et al., 2002).

Os efeitos colaterais mais importantes são: ganho de peso e hiperinsulinemia.

\section{Quetiapina}

Existe estudo da quetiapina em mania, mas a literatura sobre o uso de quetiapina na profilaxia do transtorno bipolar é limitada a casos clínicos e não há estudos sistemáticos (Zarate et al., 2000).

\section{Ziprasidona}

Existe evidência de que a ziprasidona é eficaz no tratamento da mania aguda. Entretanto, ainda não há sólida evidência que seja também eficaz no tratamento de manutenção (Gnanadesikan et al., 2003).

\section{Aripiprazol}

O papel do aripiprazol está bem estabelecido no tratamento da mania aguda (Keck et al., 2003); entretanto, não há na literatura estudos controlados sobre a eficácia do aripiprazol no tratamento de manutenção do transtorno bipolar (Taylor, 2003).

\section{Uso de antidepressivos}

A maioria dos estudos envolvendo o uso de antidepressivos em transtorno bipolar foca os antidepressivos tricíclicos, que têm demonstrado poder para induzir mania ou hipomania (Silverstone e Silverstone, 2004).

$\mathrm{O}$ uso de antidepressivos parece desestabilizar alguns pacientes, particularmente, pacientes com episódios mistos (Post et al., 2003). Aindução de ciclagem rápida é também associada ao uso de antidepressivos em transtorno bipolar (Kusumakar, 2002).

Os antidepressivos de primeira escolha para tratar depressão bipolar são: os ISRS e a bupropiona, como segunda escolha a venlafaxina tem sido recomendada (Sachs et al., 2000). Entretanto, não há evidências clínicas que sugiram qual estratégia é a melhor.

A informação disponível não apóia a eficácia do tratamento de manutenção para depressão bipolar com ou sem co-terapia com estabilizador do humor (Ghaemi, et al., 2001).

\section{Uso de ECT}

O uso da eletroconvulsoterapia (ECT) deve ser reservado para os pacientes que apresentam risco de suicídio e para aqueles que apresentam depressão refratária. É um tratamento muito eficaz na fase aguda, mas a necessidade de administração em hospitais e problemas de memória contra-indicam-no como primeira opção em tratamento de manutenção (Tsao, 2004).

\section{Terapia de combinação}

O racional do tratamento com várias drogas para manter os pacientes eutímicos é que somente $50 \%$ dos pacientes respondem a uma droga apenas e que pacientes bipolares freqüentemente apresentam comorbidades. Assim, múltiplos mecanismos de ação proporcionam melhor controle do quadro clínico e são consistentes com outros enfoques usados para tratar outras patologias como hipertensão, câncer e HIV (Biederman et al., 1998; Findling et al., 2001).

Foi demonstrado que o paciente bipolar usa em média 4,2 medicações (Sachs e Thase, 2000). Assim, a monoterapia no transtorno bipolar é exceção ao invés de regra.

Drogas combinadas podem permitir doses menores e, portanto, melhor toleradas de ambos os medicamentos. Entretanto, os perfis podem ser complementares (lítio ou valproato mais lamotrigina).

Algumas drogas podem ter efeitos colaterais aditivos como, por exemplo, olanzapina e valproato (ganho de peso).

Lítio + Antipsicótico - O lítio tem sido usado juntamente com antipsicótico em terapia de manutenção (Small et al., 1995).

Lítio + Carbamazepina - Esta combinação é usada intensamente e parece segura e eficaz, especialmente no ciclo rápido (Small et al., 1995).

Lítio + Fluoxetina - Esta combinação parece prevenir a depressão em pacientes bipolares (Tondo et al., 1997).

Lítio + Lamotrigina - Relatos de caso indicam que esta pode ser uma combinação útil (Calabrese et al., 1996).

Valproato + Carbamazepina - Houve diversos relatos da eficácia, por exemplo, quando o valproato foi adicionado aos pacientes que não respondiam à carbamazepina (Schaff et al., 1993).

\section{Tratamento de manutenção na gravidez}

O lítio pode causar anomalia de Ebstein. Uma metanálise detectou a proporção de $0.05 \%$, ou seja, risco relativo 10 a 20 vezes maior que a população em geral (Cohen et al., 1994). O risco de espinha bífida com o uso de carbamazepina $(0,5 \%$ a $1,0 \%)$ e valproato $(3 \%$ a 8\%) em mulheres em idade reprodutiva é observado (Holmes et al., 2002; Viguera et al., 2002). 


\section{Diretrizes}

O nível de recomendação do Guideline para tratamento da APA (American Psychiatric Association, 2002) é classificado em níveis (I - sólida evidência, II - evidência moderada, III - recomendação feita baseada em circunstâncias individuais). Assim, as seguintes recomendações existem para o tratamento de manutenção do transtorno bipolar:

- após um episódio de mania (I)

- em bipolar II (II)

- uso de lítio e valproato (I)

- uso de lamotrigina e carbamazepina (II)

- tratamento deve ser continuado para tratar episódios agudos (I)

- intervenções psicossociais podem ser acrescentadas (I)

Se um quadro depressivo emergir, as seguintes condutas poderão ser tomadas (APA - Guideline, 2002):

- depressão leve ou moderada - otimizar o tratamento com estabilizador de humor ou adicionar um segundo estabilizador/ antidepressivo;

- depressão com ciclagem rápida - evitar antidepressivos, estabilizador em uso mais segundo (preferencialmente lítio ou valproato) ou ainda lamotrigina.

- depressão refratária-ECT.

Segundo o Expert Consensus Guideline Series (Sachs et al., 2000) as seguintes recomendações são feitas:

- usar um estabilizador de humor em todas as fases do tratamento;

- quando um antipsicótico for necessário, os atípicos devem ser preferidos para o tratamento;

- depressões leves devem ser tratadas com um estabilizador de humor em monoterapia;
- depressão grave deve ser tratada com um antidepressivo mais um estabilizador do humor;

- mania ou depressão com ciclos rápidos deve ser tratada inicialmente com apenas um estabilizador de humor, de preferência o divalproato.

A Associação Britânica de Psicofarmacologia (Goodwin, 2003) elaborou também um consenso para tratamento do transtorno bipolar, que é semelhante ao da APA. Faz a inclusão da olanzapina como medicamento de manutenção, sendo esta mais eficaz na prevenção de episódios maníacos que depressivos.

\section{Conclusão}

Qualquer conclusão deve ser feita com cuidado, em virtude da limitada evidência disponível, com poucos estudos controlados disponíveis, número reduzido de pacientes em cada estudo, desfecho medido por resposta, ao invés de remissão, não-distinção entre recaída e recorrência e freqüentemente o uso de hospitalização como único critério de desfecho clínico.

Como recomendações gerais, caso seja necessário realizar mudanças na medicação para que o paciente continue eutímico, fazer uma mudança de cada vez, fazer avaliações sistemáticas, fazer suposições razoáveis como associar duas drogas de classes diferentes do que duas drogas de uma mesma classe. Prioridade em qualquer tratamento de manutenção deve ser dada aos fatores do paciente, como minimizar efeitos colaterais e maximizar adesão.

Em resumo, as drogas que têm demonstrado evidência sólida no tratamento de manutenção do transtorno bipolar em ordem decrescente de evidência são: lítio, valproato, carbamazepina, olanzapina e lamotrigina (esta última para depressão bipolar).

Referências bibliográficas

American Psychiatric Association - Practice Guideline for the Treatment of Patients with Bipolar Disorder. 2nd. APA, Washington, DC, 2002.

BAKER, J.P. - Lithium Discontinuation: a Meta-analysis. Lithium 5: 187-192, 1994.

Bhana, N.; Perry, C.M. - Olanzapine: a Review of its Use in the Treatment of Bipolar I Disorder. CNS Drugs 15: 871-904, 2001.

Biederman, J.; Mick, E.; Bostic, J.Q. et al. - The Naturalistic Course of Pharmacologic Treatment of Children with Maniclike Symptoms: a Systematic Chart Review. J Clin Psychiatry 59: 628-637, 1998.
Bowden, CL.; Swann, A.C.; Calabrese, J.R. et al. - Maintenance Clinical Trials in Bipolar Disorder: Design Implications of the Divalproex-Lithium-Placebo Study. Psychopharmacol Bul. 33(4): 693-639, 1997.

Bowden, C.L.; Calabrese, J.R.; McElroy, S.L. et al. - A Randomized, Placebo-controlled 12-Month Trial of Divalproex and Lithium in Treatment of Outpatients with Bipolar I Disorder. Divalproex Maintenance Study Group. Arch Gen Psychiatry 57: 481-489, 2000.

Bowden, C.L.; Calabrese, J.R., SAChs, G. et al and Lamictal 606 Study Group. - A Placebo-controlled 18-Month Trial of 
Lamotrigine and Lithium Maintenance Treatment in Recently Manic or Hypomanic Patients with Bipolar I Disorder. Arch Gen Psychiatry 60: 392-400, 2003.

Burgess, S.; Geddes, J.; Hawton, K. et al. - Lithium Maintenance Treatment of Mood Disorders. Cochrane Database Syst $\operatorname{Rev}(3)$ : CD003013, 2001.

Calabrese, J.R.; Fatemi, S.H.; WoyshVille, M.J. - Antidepressant Effects of Lamotrigine in Rapid Cycling Bipolar Disorder. Am J Psychiatry 153(9): 1236, 1996.

Calabrese, J.R.; Bowden, C.L.; Sachs, G.S. et al. - A Double-blind Placebo-controlled Study of Lamotrigine Monotherapy in Outpatients with Bipolar I Depression. Lamictal 602 Study Group. J Clin Psychiatry 60: 79-88, 1999.

Calabrese, J.R.; Suppes, T.; Bowden, C.L. et al. - A double-Blind, Placebo-controlled, Prophylaxis Study of Lamotrigine in Rapid-Cycling Bipolar Disorder. Lamictal 614 Study Group. J Clin Psychiatry 61: 841-850, 2000.

ChengapPa, K.N.; Gershon, S.; Levine, J. - The Evolving Role of Topiramate Among Other Mood Stabilizers in the Management of Bipolar Disorder. Bipolar Disord 3:215232, 2001.

Cohen, L.S.; Friedman, J.M.; Jefferson, J.W. et al. - A Reevaluation of Risk of in Utero Exposure to Lithium. JAMA 12; 271: 146-150, 1994.

Colom, F.; Vieta, E.; Martinez-Aran, A. et al. - A Randomized Triao on the Efficacy of Group Psychoeducation in the Prophylaxis of Recurrences in Bipolar Patients Whose Disease is Remission. Arch Gen Psychiatry 60: 402-407, 2003.

CoXheAd, N.; Silverstone, T.; Cookson, J. - Carbamazepine versus Lithium in the Prophylaxis of Bipolar Affective Disorder. Acta Psychiatr Scand 85: 114-118, 1992.

Davis, J.M.; JanICAK, P.G.; Hogan, D.M. - Mood Stabilizers in the Prevention of Recurrent Affective Disorders: a MetaAnalysis. Acta Psychiatr Scand 100: 406-417, 1999.

El-MallakH, R.B. - An Open Study of Methylphenidate in bipolar depression. Disorders Bipolares 2: 56-59, 2000.

Ennst, C.L.; Goldberg, J.F. - Antidepressant Properties of Anticonvulsant Drugs for Bipolar Disorder. J Clin Psychopharmacol 23: 182-192, 2003.

Esparon, J.; Kolloorl, J.; NAyLOR, G.J. et al. - Comparison of the Prophylactic Action of Flupenthixol with Placebo in Lithium Treated Manic-depressive Patients. $\mathrm{Br} \mathrm{J}$ Psychiatry 148: 723-725, 1986.

FagiolinI, A.; KuPfer, D.J.; Houck, P.R. et al. - Obesity as a Correlate of outcome in Patients with Bipolar I Disorder. Am J Psychiatry 160: 112-117, 2003.

Finding, R.L.; Gracious, B.L.; McNamara, N.K. et al. - Rapid, Continuous Cycling and Psychiatric Co-Morbidity in Pediatric Bipolar I Disorder. Bipolar Disord 3: 202-210, 2001.

GhaemI, S.N.; SACHS, G.S. - Long-Term Risperidone Treatmenti in Bipolar Disorder: 6-Month Follow Up. Int Clin Psychopharmacol 12: 333-338, 1997.

Ghaemi, S.N.; Katzow, J.J.; Desal, S.P. et al. - Gabapentin Treatment of Mood Disorders: a Preliminary Study. $J$ Clin Psychiatry 59: 426-429, 1998.
Ghaemi, S.N.; Lenox, M.S.; Baldessarini, R.J. - Effectiveness and Safety of Long-term Antidepressant Treatment in Bipolar Disorder. J Clin Psychiatry 62: 565-569, 2001.

GhaemI, S.N.; Manwani, S.G.; Katzow, J.J. et al. - Topiramate Treatment of Bipolar Spectrum Disorders: a Retrospective Chart Review. Ann Clin Psychiatry 13: 185189, 2001.

Giannini, A.J.; Taraszewski, R.; Loiselle, R.H. - Verapamil and Lithium in Maintenance Therapy of Manic Patients. $J$ Clin Pharmacol 27: 980-982, 1987.

Goldberg, J.F.; Frye, M.A.; Dunn, R.T. - Pramipexole in Refractory Bipolar Depression. Am J Psychiatry 156: 798, 1999.

Gnanadesikan, M.; Freeman, M.P.; Gelenberg, A.J. - Alternatives to Lithium and Divalproex in the Maintenance Treatment of Bipolar Disorder. Bipolar Disord 5: 203-216, 2003.

Goodwin, G.M.; SouzA, F.G.; Austin, M.P. - Lithium Augmentation in Antidepressant-resistant Patients. Br J Psychiatry 161: 128-129, 1992.

GoodwIN, G.M. - Consensus Group of the British Association for Psychopharmacology. Evidence-based Guidelines for Treating Bipolar Disorder: Recommendations From the British Association for Psychopharmacology. J Psychopharmacol 17(2): 149-173, 2003.

HoLmes, L.B. - Teratogen-induced Limb Defects. Am J Med Genet 15; 112(3): 297-303, 2002.

Jamıson, K.R. - Suicide and Bipolar Disorder. J Clin Psychiatry 61(Suppl 9): 47-51, 2000.

KeCK Jr., P.E.; McElroy, S.L.; StrakowskI, S.M. - Anticonvulsants and Antipsychotics in the Treatment of Bipolar Disorder. J Clin Psychiatry 59(Suppl 6): 74-81, 1998.

Keck Jr., P.E.; Marcus, R.; Tourkodimitris, S. et al. - Placebo Controlled, Double Blind Study of the Efficacy and Safety of Aripripazole in Patients with Acute Mania. Aripripazole Study Group. Am J Psychiatry 160: 16511658, 2003.

Kleindienst, N.; Greil, W. - Inter-episodic Morbidity and Drop-out under Carbamazepine and Lithium in the Maintenance Treatment of Bipolar Disorder. Psychol Med 32(3): 493-501, 2002.

Kusumakar, V. - Antidepressants and Antipsychotics in the Long-term Treatment of Bipolar Disorder. J Clin Psychiatr 63 (Suppl 10): 23-28, 2002.

Letterman, L.; MarkowItZ, J.S. - Gabapentin: a Review of Published Experience in the Treatment of Bipolar Disorder and Other Psychiatric Conditions. Pharmacotherapy 19(5): 565-572, 1999.

Lund, B.C.; PerRy, P.J. - Olanzapine: an Atypical Antipsychotic for Schizophrenia. Expert Opin Pharmacother 1(2): 305323, 2000.

Lusznat, R.M.; MurPhy, D.P.; Nunn, C.M. - Carbamazepine versus Lithium in the Treatment and Prophylaxis of Mania. $\mathrm{Br} \mathrm{J}$ Psychiatry 153: 198-204, 1988.

MikLowIZ, D.J.;GoLdSTEIN, M.J. - BipolarDisorder. a Family-Focused Treatment Approach. Guilford Press, New York, 1997.

Miklowitz, D.J.; Simoneau, T.L.; George, E.L. et al. - FamilyFocused Treatment of Bipolar Disorder: 1-year Effects 
of a Psychoeducational Program in Conjunction with Pharmacotherapy. Biol Psychiatry 15(48): 582-592, 2000.

Muller-Oerlinghausen, B. - Arguments for the Specificity of the Antisuicidal Effect of Lithium. Eur Arch Psychiatry Clin Neurosci 251(Suppl 2): 72-75, 2001.

Obrocea, G.V.; Dunn, R.M.; Frye, M.A. et al. - Clinical Predictors of Response to Lamotrigine and Gabapentin Monotherapy in Refractory Affective Disorders. Biol Psychiatry 5: 253260, 2002.

Placidi, G.F.; LenzI, A.; LazzerinI, F. et al. - The Comparative Efficacy and Safety of Carbamazepine versus Lithium: a Randomized, Double-blind 3-year Trial in 83 Patients. J Clin Psychiatry 47: 490-544, 1986.

Perry, A.; Tarrier, N.; Morriss, R. et al. - Randomised Controlled Trial of Efficacy of Teaching Patients with Bipolar Disorder to Identify Early Symptoms of Relapse and Obtain Treatment. BMJ 318(7177): 149-153, 1999.

Post, RM.; LeVerich, G.S.; Rosoff, A.S. et al. - Carbamazepine Prophylaxis in Refractory Affective Disorders: a Focus on Long-term Follow-Up. J Clin Psychopharmacol 10: 318-327, 1990.

Post, R.M.; Baldassano, C.F.; Perlis, R.H. et al. - Treatment of Bipolar Depression. CNS Spectr 8(12): 1-10, 2003.

RothваUм, B.0.; Astin, M.C. - Integration of Pharmacotherapy and Psychotherapy for Bipolar Disorder. J Clin Psychiatry 61(Suppl 9): 68-75, 2000.

SACHs, G.S.; ThASE, M.E. - Bipolar Disorder Therapeutics: Maintenance Treatment. Biol Psychiatry 15; 48(6): 573581, 2000.

Sachs, G.S.; Printz, D.J.; Kahn, D.A. et al. - The Expert Consensus Guideline Series: Medication Treatment of Bipolar Disorder 2000. Postgrad Med 1-104, 2000.

SchafF, M.R.; FawCett, J.; ZajeckA, J.M. - Divalproex Sodium in the Treatment of Refractory Affective Disorders. J Clin Psychiatry 54: 380-384, 1993.

Schaffer, C.B.; SChafFer, L.C. - Gabapentin in the Treatment of Bipolar Disorder. Am J Psychiatry. 154: 291-292, 1997.

Schaffer, L.C.; Schaffer, C.B. - Tiagabine and the Treatment of Refractory Bipolar Disorder. Am J Psychiatry 156: 20142015, 1999.

Scott, J.; Garland, A.; Moorhead, S. - A Pilot Study of Cognitive Therapy in Bipolar Disorders. Psychol Med 31: 459-67, 2001.
Silverstone, P.H.; Silverstone, T. - A Review of Acute Treatments for Bipolar Depression. Int Clin Psychopharmacol 19: 113124, 2004.

SmalL, J.G.; Klapper, M.H.; MarhenKE, J.D. et al. - Lithium Combined with Carbamazepine or Haloperidol in the Treatment of Mania. Psychopharmacol Bull 31: 265-272, 1995.

Stoll, A.L.; Severus, W.E.; Freeman, M.P. et al. - Omega 3 Fatty Acids in Bipolar Disorder: a Preliminary Double-Blind, Placebo-controlled Trial. Arch Gen Psychiatry 56: 407412, 1999.

Suppes, T.; WebB, A.; Paul, B. et al. - Clinical Outcome in a Randomized 1-Year Trial of Clozapine Versus Treatment as Usual for Patients with Treatment-Resistant Illness and a History of Mania. Am J Psychiatry 156: 1164-1169, 1999.

TAYLOR, D.M. - Aripiprazole: a Review of its Pharmacology and Clinical Use. Int J Clin Pract 57: 49-54, 2003.

Tohen Jr., M.; Zarate, C.A. - Antipsychotic Agents and Bipolar Disorder. J Clin Psychiatry 59(Suppl 1): 38-48, 1998.

ToHen, M.F. et al. - Olanzapine-Fluoxetine Combination for Phychotic Major Depression. Presented at $155^{\text {th }}$ American Psychiatric Association Annual Meeting 1823, Philadelphia, Pa, 2002.

Tondo, L.; Jamison, K.R.; BaldessarinI, R.J. - Effect of Lithium Maintenance on Suicidal Behavior in Major Mood Disorders. Ann N Y Acad Sci 836: 339-351, 1997.

Tondo, L.; Hennen, J.; BaldessaRinI, R.J. - Lower Suicide Risk With Long-term Lithium Treatment in Major Affective Illness: a Meta-analysis. Acta Psychiatr Scand 104: 163-172, 2001.

TSAO, C.I.; JaIn, S.; GIBSON, R.H. et al. - Maintenance ECT for Recurrent Medication-refractory Mania. J ECT 20: 118119, 2004.

Viguera, A.C.; Cohen, L.S.; Baldessarinı, R.J.; Nonacs, R. - Managing Bipolar Disorder During Pregnancy: Weighing the Risk and Benefits. Can J Psychiatry 47: 426-36, 2002.

Wations, S.E.; Callender, K.; Thomas, D.R. et al. - The effect of Carbamazepine and Lithium on Remission from Affective Illness. Br J Psychiatry 150: 180-2, 1987.

Zarate JR., C.A.; Rothschild, A.; Fletcher, K.E. et al. - Clinical Predictors of Acute Response With Quetiapine in Psychotic Mood Disorders. J CLin Psychiatry61(3): 1859, 2000.

Zarate JR., C.A.; Tohen, M.; Baldessarinı, R.J. - Clozapine in Severe Mood Disorders. J Clin Psychiatry 56: 411-417, 1995. 